\title{
Science and Adaptive Optics Requirements of MICADO, the E-ELT adaptive optics imaging camera
}

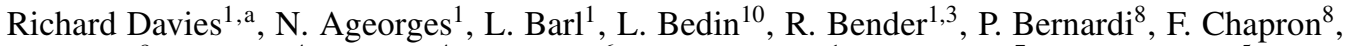 \\ Y. Clenet ${ }^{8}$, A. Deep ${ }^{4}$, E. Deul ${ }^{4}$, M. Drost ${ }^{6}$, F. Eisenhauer ${ }^{1}$, R. Falomo ${ }^{7}$, G. Fiorentino ${ }^{5}$, \\ N. M. Förster Schreiber ${ }^{1}$, E. Gendron ${ }^{8}$, R. Genzel ${ }^{1}$, D. Gratadour ${ }^{8}$, L. Greggio ${ }^{7}$, F. Grupp ${ }^{3}$, E. Held ${ }^{7}$, \\ T. Herbst ${ }^{2}$, H.-J. Hess ${ }^{3}$, Z. Hubert ${ }^{8}$, K. Jahnke ${ }^{2}$, K. Kuijken ${ }^{4}$, D. Lutz ${ }^{1}$, D. Magrin ${ }^{7}$, B. Muschielok ${ }^{3}$, \\ R. Navarro ${ }^{6}$, E. Noyola ${ }^{1}$, T. Paumard ${ }^{8}$, G. Piotto ${ }^{7}$, R. Ragazzoni ${ }^{7}$, A. Renzini ${ }^{7}$, G. Rousset ${ }^{8}$,

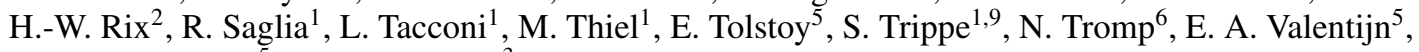 \\ G. Verdoes Kleijn ${ }^{5}$, and M. Wegner ${ }^{3}$
}

1 Max Planck Institute for extraterrestrial Physics, Germany

2 Max Planck Institute for Astronomy, Germany

3 Munich University Observatory, Germany

4 University of Leiden, Netherlands

5 University of Groningen, Netherlands

6 NOVA Optical/IR Instrumentation Group, Netherlands

7 Astronomical Observatory of Padova, INAF, Italy

8 Laboratory of Space Studies and Instrumentation in Astrophysics, Observatory of Paris, France

9 Institute for Millimetre Radio Astronomy, France

10 Space Telescope Science Institute, USA

\begin{abstract}
MICADO is the adaptive optics imaging camera being studied for the E-ELT. Its design has been optimised for use with MCAO, but will have its own SCAO module for the initial operational phase; and in principle could also be used with GLAO or LTAO. In this contribution, we outline a few of the science drivers for MICADO and show how these have shaped its design. The science drivers have led to a number of requirements on the AO system related to astrometry, photometry, and PSF uniformity. We discuss why these requirements have arisen and what might be done about them.
\end{abstract}

\section{MICADO Overview}

MICADO is the Multi-AO Imaging Camera for Deep Observations, which is being designed to work with adaptive optics on the 42-m European Extremely Large Telescope. During the Phase A study, the instrument has been optimised for the multi-conjugate adaptive optics module MAORY [1]; but it is also able to work with other adaptive optics systems. In particular, it includes a separate module to provide a single conjugate adaptive optics capability with natural guide stars during the early operational phase. Combined with the simplicity and robustness of the camera design, which have been borne in mind throughout its development, this has led the consortium to view MICADO as an ideal E-ELT first light instrument.

The instrument is very compact and is supported underneath the AO systems so that it rotates in a gravity invariant orientation, as shown in Fig. 1. It is able to image, through a large number of selected wide and narrow band near infrared filters, a wide 53" field of view at the diffraction limit of the E-ELT. In addition to a high throughput camera with a fixed 3 mas pixel scale, MICADO will have an auxiliary arm to provide an increased degree of flexibility. In the current design, this arm provides (i) a finer 1.5 mas pixel scale over a smaller field, and (ii) a 4 mas pixel scale for a simple, medium resolution longslit spectroscopic capability. However, in principle the auxiliary arm also opens the door to many other options, including simple polarimetry, a 'dual imager' based on a Fabry-Perot etalon

\footnotetext{
a e-mail: davies@mpe.mpg.de
} 


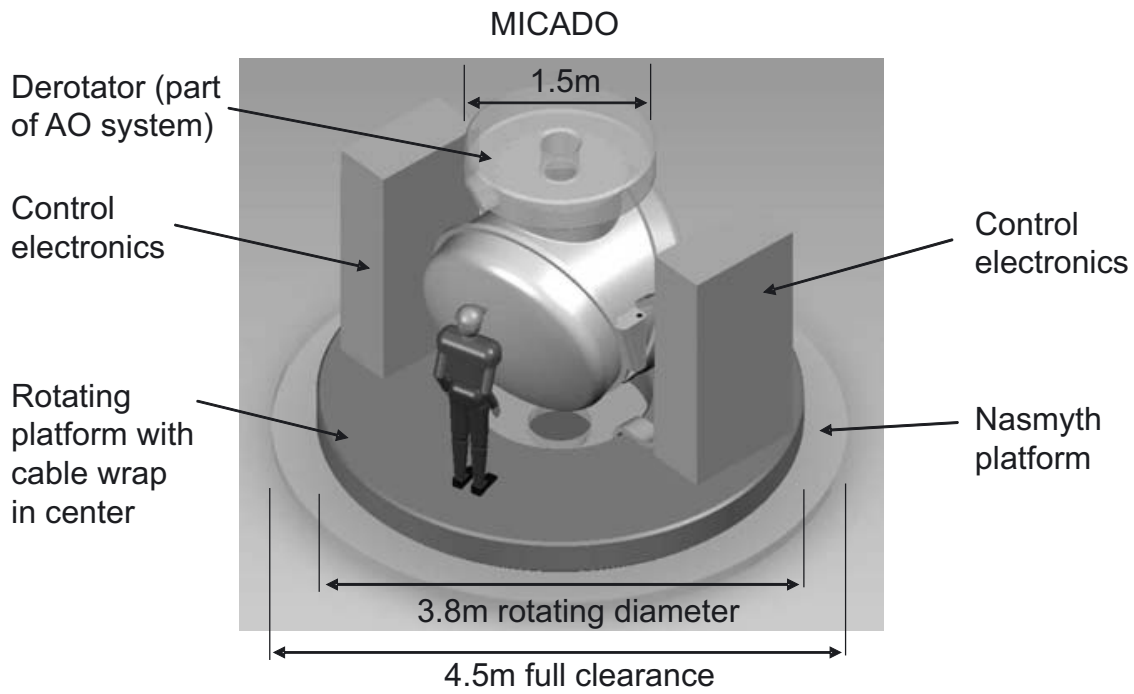

Fig. 1. View of MICADO as it will hang underneath the AO systems. The cryostat is less than $2 \mathrm{~m}$ across, and all key components on the inside can be accessed through two large doors without dismounting the instrument. The electronics will be in two cabinets on a co-rotating platform, which also houses the cable-wrap and itself sits directly on the Nasmyth platform.

to image separate emission line and continuum wavelengths simultaneously, or a high time resolution detector.

\section{MICADO Key Capabilities}

Early in the project, the consortium highlighted several key capabilities that exemplify the unique features of the E-ELT, and at which MICADO on the E-ELT will excel in comparison to other facilities. These are at the root of the science cases [2] and have driven the design of the camera.

Sensitivity and Resolution. Its high quality optics and 3 mas pixels mean MICADO is optimised for imaging at the diffraction limit, and will fully sample the 6-10 mas FWHM in the J-K bands. With a throughput exceeding $60 \%$ its sensitivity at $1-2 \mu \mathrm{m}$ will, for the AO performance predicted by MAORY, be comparable to, and may even surpass, JWST. But MICADO's superior resolution means that it will be able to probe the detailed structure of objects that are unresolved by JWST. In addition, its field of view of nearly 1 arcmin yields a significant multiplex advantage compared to other groundbased cameras such as IRIS on the TMT. Together, these characteristics make MICADO a powerful tool for many science cases, from studies of faint high redshift galaxies to performing photometry in crowded fields. This latter topic is discussed in more detail in Sec. 4.

Precision Astrometry. The primary imaging field of MICADO employs a catoptric design using only fixed mirrors. Together with the gravity invariant rotation and the baseline to use Hawaii-4RG detectors (developed to meet the stringent requirements of space astrometry missions), this makes MI$\mathrm{CADO}$ an ideal instrument for astrometry. A robust pipeline, based on software already available in the AstroWISE system, will bring precision astrometry into the mainstream. An analysis of the statistical and systematic effects [3] shows that proper motions of $40 \mu \mathrm{as} / \mathrm{yr}$ in a single epoch of observations should be achievable. At this level, many novel science cases become feasible, and a few of these are discussed in Sec. 3.

High Throughput Spectroscopy. The obvious complement to plain imaging is spectroscopy, and this capability is mandatory if MICADO is to be considered as a first light instrument. We have imple- 
mented a simple slit spectrometer with a high throughput that is ideal for obtaining spectra of compact objects. The resolution of $R \sim 3000$ is sufficient to probe between the near infrared $\mathrm{OH}$ lines. This simple addition will enhance many science cases, for example: deriving stellar types and 3D orbits in the Galactic Center; using velocities of stars in nearby galaxies to probe central black hole masses and extended mass distributions; measuring absorption lines in galaxies at $z=2-3$ and emission lines in galaxies at $z=4-6$ to derive their ages, metallicities, and star forming histories; and obtaining spectra of the first supernovae at $z=1-6$.

Simple, Robust, Available Early. Imaging is fundamental to astronomy, and MICADO is designed to excel at this technique. By doing so it will both exploit and promote the most unique features of the E-ELT. The instrument's optical and mechanical simplicity lead directly to the stability needed for astrometry and photometry; and in addition mean that the instrument can be available for first light. In order to capitalize on this potential, MICADO has been designed to work not only with MCAO, but also has its own robust SCAO system that will provide wavefront sensing with NGS to control the telescope's deformable and tip-tilt mirrors.

\section{Astrometry}

With an astrometrical precision of $40 \mu$ as and proper motions of $10 \mu \mathrm{as} / \mathrm{yr}$ detectable after only a few years, one finds that 'everything moves' and many remarkable science cases become feasible. MICADO will be able to measure stellar orbits not only in the Galactic Center, but also around other supermassive black holes in nearby galaxies. The proper motions of globular clusters can be used to derive their distance via parallax displacement, separate their stellar populations from field stars, and, by looking for kinematic families, probe the formation and evolution of the Galaxy. By analysing the internal kinematics of dwarf spheroidals to derive their anisotropy and mass density profiles, one can test hierarchical models of structure formation. Perhaps one of the most fascinating topics that can be resolved is that of intermediate mass black holes.

\subsection{Intermediate Mass Black Holes}

Black holes with masses of order $10^{3}-10^{4} \mathrm{M}_{\odot}$ are expected theoretically from simulations of massive dense star clusters, such as the Arches cluster [4] and IRS 13 [5] near the centre of the Galaxy. In the most massive globular cluster Omega-Cen, the mass has been measured as $4 \times 10^{4} \mathrm{M}_{\odot}$ [6]. However, recently doubts have been cast on this result from astrometric data, which have been use to derive proper motion dispersions along both the radial and tangential directions. They have revealed a small but significant degree of anisotropy [7,8]. For a luminosity profile with a shallow cusp, the authors found $\mathrm{M}_{\mathrm{BH}} \sim 2 \times 10^{4} \mathrm{M}_{\odot}$; but remarkably their model with a core profile was consistent with no black hole. Thus these authors could only put an upper limit on the black hole mass, and the question of whether Omega-Cen contains a black hole is once again open.

This result was based on 4 years of HST imaging which yielded an astrometric precision of about $100 \mu$ as. MICADO will be able to achieve better precision in a single epoch, and hence opens the way to probing the anisotropy and black hole masses in this and many other globular clusters.

\subsection{Statistical and Systematic Effects}

There are many effects that have to be taken into consideration in the instrument design, and carefully calibrated, in order to reach the astrometric precision required [3]. Of these, 3 are relevant to adaptive optics correction. Differential tilt jitter is the small residual motion of objects in the focal plane, and although an order of magnitude smaller for MCAO than for SCAO, is still significant. Because it is a statistical effect, it has to be integrated out, although more advanced methods of combining frames can lead to better results [9]. Both instrumental and atmospheric effects can influence the NGS used 


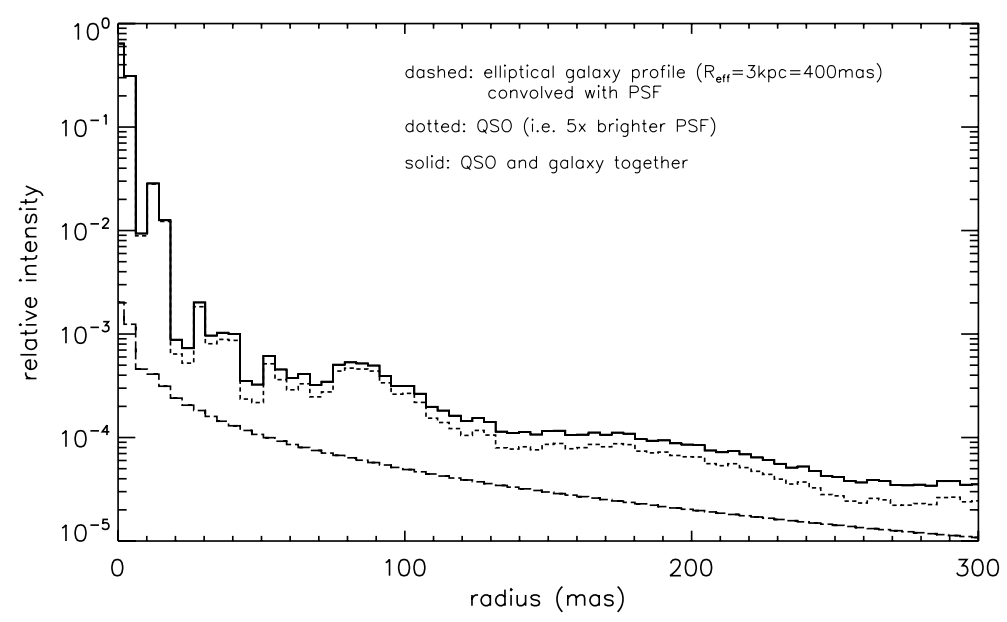

Fig. 2. Illustration of a QSO and its host galaxy at high redshift $(z>2)$, showing the similarity between the radial profiles of the two components; and hence that in order to subtract the QSO contribution to reveal the underlying host galaxy, one needs to know the PSF well (a simple Strehl ratio is insufficient: a detailed profile is required).

for tip-tilt sensing. For example, if rotation of the instrument is inexact, the NGS will appear to move in different directions. Alternatively both achromatic refraction (which arises because opposite sides of the field of view are at different airmass) and chromatic refraction (which depends on the colour of the NGS) will also cause the NGS to drift slowly. As the AO system corrects for these shifts, it will warp the field of view. If the warping is, as expected, only a low order effect, it can easily be calibrated out in individual frames. On the other hand, it may be possible to avoid it completely by updating the centering position of the NGS on their detectors to follow their time-averaged barycentres. Spatial variations and asymmetries in the PSF could also lead to astrometric inaccuracies. This is harder to quantify without detailed and time comsuming PSF simulations; but work on preliminary analytical PSFs by the MAORY consortium [1] suggests that it should have little impact. The importance and impact of PSF knowledge - or lack of it - is discussed in the next section.

\section{PSF and Photometry}

Most science cases require at least some knowledge of the PSF. In the following subsections we look at 2 highly contrasting science cases that both require a good knowledge of the PSF, but apply it in different ways.

\subsection{QSO Host Galaxies}

Some of the key issues in galaxy evolution concern the relation between the mass of a supermassive black hole in the centre of a galaxy and that of the stellar spheroid surrounding it, the mechanisms that regulate their respective growth, and the rapid truncation of star formation that leads to the observed colour bimodality. In our current understanding, it is AGN feedback that is responsible for all these. As such, QSOs and their host galaxies represent a crucial piece of the puzzle, and studying these to high redshift is an important goal. However, measuring the properties of the host galaxy requires one to subtract the central QSO, which is often several to tens of times brighter - a task that is increasingly difficult at higher redshifts, and requires a good estimate of the PSF [10]. This is illustrated in Fig. 2, which shows the radial profile one might expect for an elliptical host galaxy, the radial profile of the central QSO (i.e. a PSF), and the small difference between them. Approximately, for a QSO that is 5 
Observations of Omega-Cen

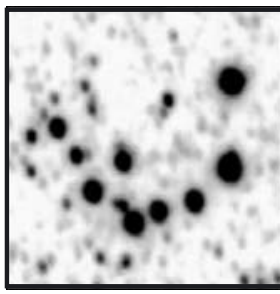

ISAAC

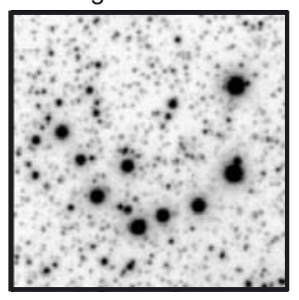

MAD

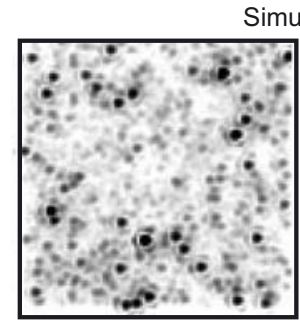

JWST
Simulations

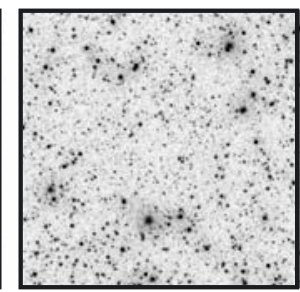

MICADO

Fig. 3. Examples showing how, by reducing crowding, enhanced resolution can increase the effective sensitivity. Left: observations of Omega-Cen by ISAAC and MAD at the VLT. Right: simulations of a crowded field as seen by JWST and MICADO. In crowded fields, MICADO's higher resolution will enable it to reach several magnitudes deeper than JWST.

times brighter than the host galaxy, an error of only $2 \%$ in the estimation of the Strehl ratio leads to an uncertainty of $10 \%$ in the host galaxy magnitude. And if the error on the Strehl is as much as $20 \%$, then one might not even detect the host galaxy at all.

From the astronomer's perspective, for science cases such as this, reconstruction of the on-axis PSF from WFS data would the ideal solution. Yet good PSF reconstruction, specifically when one or more LGS are used in the wavefront sensing, remains a challenge. In this respect the large field of view of MICADO is an asset even though the science target itself is compact, because it becomes highly likely that suitable empirical PSFs can be found within the field even at high galactic latitudes. If the PSFs do not vary much across the field, they can be combined and used directly. Alternatively if one can parametrize several PSFs in different locations it may be possible to interpolate the PSF at the position of the QSO, or indeed at any other point in the field. A positive step in addressing this by developing a simple model for the PSF has been taken by the MAORY consortium [1].

\subsection{Resolved Stellar Populations}

An alternative way to study galaxy evolution is through the relic populations in nearby galaxies [11]. Accurate photometry of spatially resolved stellar populations enables one to create colour-magnitude diagrams in order to derive a galaxy's star formation history. The ultimate goal of such work is to apply these methods to galaxies in the Virgo cluster which, at a distance of $\sim 17 \mathrm{Mpc}$, is the nearest large cluster, with over 2000 member galaxies of all morphological types. Directly counting the number of stars on different branches enables one to derive a coarse but robust star formation history for a galaxy that, by probing the oldest stars with ages $>10^{10} \mathrm{Gyr}$, can be traced back to the early universe [12]. In the Leo Group (at a distance of $\sim 11 \mathrm{Mpc}$ ), MICADO can comfortably reach the Horizontal Branch. In closer galaxies, such as Cen A at $\sim 3 \mathrm{Mpc}$, it will be possible to measure the entire Red Giant Branch (RGB) down to the level of the main sequence turnoff, and hence to address the problem of the age-metallicity degeneracy.

Although sensitivity is important in such work, the crucial aspect is resolution since this allows one to probe ever more crowded fields, as illustrated by Fig. 3. Observations with ISAAC and MAD at the VLT of the sme stellar field have shown that the improvement in resolution from $0.6^{\prime \prime}$ to $0.1^{\prime \prime}$ leads to 3 mag greater depth [13,14]: by reducing the crowding, better resolution yields an effective gain in sensitivity. The factor 6 in resolution between ISAAC and MAD is similar to the difference between JWST and MICADO, and so we also expect to reach several magnitudes deeper in crowded fields. It means that, in the Virgo cluster, rather than probing the fringes of galaxies at $3-4 R_{\text {eff }}$, we will be able to work near the centres of the galaxies at $1-2 R_{\text {eff }}$. This is a fundamental issue, since it enables one to study the regions of galaxies where a large fraction of the stellar mass is located, and hence gain significant information about the global star formation history. In addition it will be possible to address the problem of stellar population gradients in giant galaxies using photometry of individual stars, rather than integrated light profiles. 
Despite the science field being full of stars, one still needs to be careful about extracting the PSF from the highly crowded data. A number of widely used programs already exist to do this, such as DAOPHOT and StarFinder, and these appear to work well also on simulated MICADO frames. But one aspect that needs to be assessed further is the impact on photometric accuracy of spatial variations across the large field. Simulations show that if these are not taken into account, the photometric scatter increases markedly. However, one possible solution is to make use of the fact that in MICADO the field is imaged onto an array of 16 detectors, where each detector subtends an angle of only $12.3^{\prime \prime}$. And on any single detector, PSF variations will be negligible. Thus, until spatially variant PSFs can be dealt with in photometry packages, an interim way to cope with them is simply to treat each detector independently.

\section{Conclusion}

MICADO is the adaptive optics imaging camera being studied at Phase A for the E-ELT. It has been designed to be simple, robust, and could be ready for the E-ELT first light. The primary imaging field has a high throughput, reflective, gravity invariant design using only fixed mirrors, making it ideally suited to photometric and astrometric science applications. But in order to achieve its goals, there are a two important adaptive optics issues that need to be fully understood and resolved: (i) the impact of instrumental and atmospheric effects on the NGS, and what the knock-on effects are on field warping; (ii) how best to acquire a detailed knowledge of the PSF and its spatial variations.

\section{References}

1. Diolaiti E., et al., this proceedings

2. Renzini A., et al., MICADO Phase A Scientific Analysis Report, E-TRE-MCD-561-0007

3. Trippe S., et al., A\&A, submitted

4. Portegies Zwart S., et al., ApJ, 641, (2006) 319

5. Maillard J., et al., A\&A, 423, (2004) 155

6. Noyola E., et al., ApJ, 676, (2008) 1008

7. Anderson J., van der Marel R., ApJ, submitted

8. Van der Marel R., Andersen J., ApJ, submitted

9. Cameron P., et al., AJ, 137, (2009) 83

10. Jahnke K., Kuhlbrodt B., Wisotzki L., MNRAS, 352, (2004) 352

11. Tolstoy E., Hill V., Tosi M., ARA\&A, 47, (2009) 371

12. Greggio L., et al., Observed HR Diagrams and Stellar Evolution, ASP Conf. Ser. 274, 444

13. Marchetti E., et al., ESO Messenger, 129, (2007) 8

14. Bono G., et al., Science with the VLT in the ELT Era, (Springer, Netherlands, 2009) 67 